\title{
Audio visual speech source separation via improved context dependent association model
}

\author{
Alireza Kazemi*, Reza Boostani and Fariborz Sobhanmanesh
}

\begin{abstract}
In this paper, we exploit the non-linear relation between a speech source and its associated lip video as a source of extra information to propose an improved audio-visual speech source separation (AVSS) algorithm. The audio-visual association is modeled using a neural associator which estimates the visual lip parameters from a temporal context of acoustic observation frames. We define an objective function based on mean square error (MSE) measure between estimated and target visual parameters.

This function is minimized for estimation of the de-mixing vector/filters to separate the relevant source from linear instantaneous or time-domain convolutive mixtures. We have also proposed a hybrid criterion which uses AV coherency together with kurtosis as a non-Gaussianity measure. Experimental results are presented and compared in terms of visually relevant speech detection accuracy and output signal-to-interference ratio (SIR) of source separation. The suggested audio-visual model significantly improves relevant speech classification accuracy compared to existing GMM-based model and the proposed AVSS algorithm improves the speech separation quality compared to reference ICA- and AVSS-based methods.
\end{abstract}

Keywords: Audio-visual speech source separation; Bimodal coherency; Blind source separation; Independent component analysis

\section{Introduction}

Audio-visual speech source separation (AVSS) is a growing field of research that is developed in recent years. It is derived from mixing audio-visual speech processing (AVSP) and blind source separation (BSS) techniques.

Speech is originally a bimodal audio-visual process. Perceptual studies on human audition have revealed that visual modality has effective contributions in speech intelligibility [1], perception [2] and detection [3] especially in the noisy and multi-source (cocktail party) situations. According to the McGurk-McDonald effect [4] (that is, sensing the auditory part of a phonetic sound with visual part of another one, results in illusion of perception of a third one), it is evident that there is an early stage interaction between audio and visual stimuli in the brain. This is confirmed in [5] that early integration of audio and

*Correspondence: kazemi@cse.shirazu.ac.ir

CSE\&IT Department, Electrical and Computer Engineering School, Shiraz University, Molla-Sadra, 71348-51154 Shiraz, Iran visual modalities can help in the identification and hence enhancement of speech in noisy environment. The performance of automatic speech processing systems degrades drastically in the presence of noise or other acoustic sources. Thus, researchers have tried to incorporate visual modality to automatic speech processing systems upon the perceptual findings.

Both audio and visual modalities of speech originate from gestures and dynamics of articulators along the speaker's vocal tract. Hence, there is an intrinsic relation between these two speech cues. Although among all articulators, just the lip and, partially, jaws are visually observable. This partial observation bears a stochastic but exploitable relation between audio and visual cues.

It is inspiring to consider AV relation as two coherent and complementary components. In the automatic speech processing community, there has been early notification and interest (since 1984 [6]) for exploiting the complementary (orthogonal) portion of AV information

\section{Springer}

(c) 2014 Kazemi et al.; licensee Springer. This is an Open Access article distributed under the terms of the Creative Commons Attribution License (http://creativecommons.org/licenses/by/2.0), which permits unrestricted use, distribution, and reproduction in any medium, provided the original work is properly cited. 
prior to its coherent (non-orthogonal) portion. The complementary information of AV data is truly adopted in audio-visual speech recognition (AVSR) in either of early(feature), middle- (model), or late- (decoding) stage fusion schemes to enhance robustness against acoustic distortions. In recent years (since 2001 [7]), researchers have proposed methods based on exploiting the coherent component of AV processes for applicable tasks like speech enhancement [7-9], acoustic feature enhancement [10], visual voice activity detection (VVAD) [11], and AV source separation (AVSS) [11-24].

In [12], a statistical AV model based on Gaussian mixture models (GMMs) is presented for measuring the coherency of audio and its corresponding video and is used for extracting speech of interest from instantaneous squared mixtures on a simple French logatoms AV corpus. They have extended their method in [14] and assessed it on a more general sentence corpus and also for degenerate mixtures. Wang et al. [15] have exploited a similar GMM model (but using different AV features) as a penalty term for solving convolutive mixtures. That method seems to be inefficient because it should convert the separating system from frequency to time domain repeatedly. Rajaram et al. [13] have incorporated visual information in a Bayesian AVSS for separation of twochannel noisy mixtures. Their method adopts a Kalman filter with additional independence constraint between the states (sources). Rivet et al. [16] have adopted the AV coherency of speech (measured by a trained log-Rayleigh distribution) for resolving the permutation indeterminacy in the frequency domain separation of convolutive mixtures. They have also proposed another method [11] for convolutive AVSS based on developing a VVAD and using it in a geometric separation algorithm using sparse source assumption.

Sigg et al. in a pioneering work [17] have proposed a single microphone AVSS method by developing a nonnegative sparse canonical correlation analysis (NS-CCA) algorithm. Their method jointly separates audio signals and localizes their corresponding visual sources. Following them, Casanovas and Monaci et al. [18-21] have proposed single microphone AV separation and localization methods by sparse and redundant atomic representation of AV signals. They use cross-modal correlations between $\mathrm{AV}$ atoms as similarity measure to cluster visual atoms for localizing visual sources and then separating audio signals.

Liang et al. [22] have incorporated visual localization to improve the fast independent vector analysis (FastIVA) as a frequency domain convolutive method. They use location of sources for smart initialization of FastIVA to solve its block permutation. Liu et al. [23] have proposed an AV dictionary learning method (AVDL) and have used it for AV-BSS via bimodal sparse coding to estimate timefrequency (TF) masks.
Khan et al. [24] have proposed a video-aided separation method for two-channel reverberant recordings which estimates direction of sources via visual localization to be used in probabilistic models which are refined using EM algorithm and evaluated at discrete TF points to generate separating masks.

In this paper, we develop a visually informed speech source separation algorithm called MLP-AVSS which considers temporal dependency between consecutive AV frames. We have suggested to model AV coherency using a multi-layer perceptron (MLP) for AV association. This model with lower number of parameters can capture AV coherency significantly better relative to the GMM AV model of $[12,14,15]$. We have also proposed a hybrid measure of kurtosis and visual coherency and based on that a time domain convolutive AVSS algorithm. We have assessed quality of suggested AV model and its induced AVSS methods on two discrete (alpha-digits) and continuous (poet-verses) audio-visual corpora. The former is a corpus of Persian and English alpha digits and the later is a corpus of poem verses from about 20 Persian poets.

The rest of this paper is organized as follows: In Section 2, we briefly review BSS and AVSP background and then focus on the relevant AVSS work. Section 3 illustrates the proposed MLP-based AV model and AVSS algorithm. Section 3.3 presents a hybrid AV coherent and independent criterion, and based on that, we move toward a time-domain convolutive extension. In Section 4, audiovisual materials including AV corpus, parametrization and modeling procedures is considered. In Section 5, experimental set-up and the experimental results are illustrated and analyzed. Finally, the paper is concluded in Section 6.

\section{Background review}

AVSS has emerged from mixing BSS and audio-visual speech processing techniques [16]. In this section, after a brief review of BSS and AV speech processing background, we explain the speech separation in terms of standard source separation problem and then discuss the suggested AV separation approach as an improved solution for this problem.

\subsection{Blind source separation problem}

Commonly, a blind source separation problem is briefly defined by its forward mixing model. In this paper, we consider the problem of source separation from a linear instantaneous mixture defined as

$$
\mathbf{x}(t)=\mathbf{A}(t) \mathbf{s}(t)
$$

where $\mathbf{s}(t)=\left[s_{1}(t), \ldots, s_{N}(t)\right]^{T} \in \mathbb{R}^{N}$ is vector of source samples, $\mathbf{x}(t)=\left[x_{1}(t), \ldots, x_{M}(t)\right]^{T} \in \mathbb{R}^{M}$ is vector of mixed signals and $\mathbf{A}(t) \in \mathbb{R}^{M \times N}$ is mixing matrix at the time instance $t$. It should be noticed that both $\mathbf{s}(t)$ and A are unknown. Hence, the problem is designated to BSS 
that is estimation of unmixed signals $\mathbf{y}(t)=\mathbf{B}(t) \mathbf{x}(t)$ from mixed signals $\mathbf{x}$ using an unknown de-mixing matrix B such that they are as similar as possible to unknown sources $\mathbf{s}$.

In (1), both sources $\mathbf{s}(t)$ and the mixing process $\mathbf{A}(t)$ are considered non-stationary in time. In speech processing, sources (i.e. speech signals) are naturally non-stationary since (i) phonemes (and even sub-phonemes) have different waveform statistics and (ii) a speaker may either speak or be silent over the time. Also, the mixing model may be non-stationary in time because speakers may have motion relative to sensors (microphones). It is hard to solve the problem in this case; however, if sources and mixture can be considered piecewise stationary, a solution is to divide signals to batches and solve the BSS on each batch separately:

$$
\left\{\begin{array}{l}
\mathbf{x}_{\tau}(t)=\mathbf{A}_{\tau} \mathbf{s}_{\tau}(t) \\
\mathbf{y}_{\tau}(t)=\mathbf{B}_{\tau} \mathbf{x}_{\tau}(t)
\end{array}\right.
$$

where $\tau$ is the batch index iterating over all batches of the signals. In this case, the mixing and de-mixing models $\left(\mathbf{A}_{\tau}, \mathbf{B}_{\tau}\right)$ are time invariant during each batch. Another solution is to consider adaptive source separation techniques which is beyond the scope of this paper.

Independent component analysis (ICA) is the most wellknown family of solutions for BSS problems in which algorithms such as Infomax [25], FastICA [26] and JADE [27] (to name famous ones) try to estimate sources by adopting the statistical independence assumption. The solution of most ICA algorithms is based on optimizing their specific objective functions $J(\mathbf{B} ; \mathbf{x})$ which measure the independence via different orders of signal's statistics. De-mixing matrix is then estimated by:

$$
\mathbf{B}_{\tau}=\underset{\mathbf{B}}{\operatorname{argmin}}\left\{J\left(\mathbf{B} ; \mathbf{x}_{\tau}\right)\right\}
$$

\subsection{Audio-visual speech processing}

Before explaining AV source separation methods, it is necessary to review some issues in $\mathrm{AV}$ speech processing which also inherently arises in AV source separation:

- The speech signal and lip video are non-stationary in time.

- The rate of speech samples and video frames is significantly different. In this study, the speech signal is recorded by $F s=16,000 \mathrm{~Hz}$ while the video frame rate is $F r=30 \mathrm{fps}$.

- Speech signal and video frames have large numbers of samples (pixels) containing sparse information. This prevents creating audio-visual models directly from these signals.

To cope with first two issues, in most speech processing problems, speech is processed frame-wise with frames of $20-30$ ms length where speech signal can be considered stationary. In AV speech processing, it is convenient to choose speech frame length such that audio and video frame rates are equal.

For handling the third issue, the routine solution is to extract compact and informative acoustic and visual features from speech and video frames such that each frame is represented with a few number of parameters. Framewise processing of speech is practical in most speech processing tasks, but the amount of speech signal in a single frame may be insufficient for source separation algorithms to perform accurately. Hence, a couple of consecutive frames must be used in each batch $\tau$.

\subsection{Audio-visual source separation}

Consider problem of speech source separation in the case of instantaneous mixture of Equation (2). Most solutions (including ICA-based ones) have two major drawbacks which limit their applicability. The major problem is that ICA-based methods can estimate sources just up to a scale $\mathbf{D}$ and permutation $\mathbf{P}$

$$
\mathbf{y}_{\tau}(t)=\mathbf{D}_{\tau} \mathbf{P}_{\tau} \hat{\mathbf{s}}_{\tau}(t)
$$

that is signal's amplitude gain and their order cannot be determined using these algorithms. The permutation of estimated sources may also change within consecutive frames, because sources are non-stationary in time and space. Having true or at least stable ordering of sources is crucial in most automatic speech processing applications. Furthermore, ICA-based methods do not consider or perform weakly in case of noisy and degenerate mixtures (i.e., mixtures with $M<N$ ).

Incorporation of visual modality of speech as a source of extra information, can help to solve these problems. The permutation problem can be simply resolved and enhancement in the separation performance is gained in regular and degenerate mixtures.

Most AVSS algorithms work based on maximization of AV coherency between unmixed signals $y$ and their corresponding video streams. It is shown in [12] that given coarse spectral envelope of sources, one can solve a system of equations for calculation of de-mixing matrix in regular mixtures. Moreover there exists a stochastic coherent relation between the speech spectral envelope and the lip visual features $[9,12]$. These two facts have guided researchers toward capturing AV relation using different models and adopt it for AVSS tasks.

In [12] and [14], authors have proposed a joint statistical distribution $p_{\text {av }}(\mathcal{S}, \mathcal{V})$ as an AV model which measures the coherency between the acoustic spectral $(\mathcal{S})$ and lip visual $(\mathcal{V})$ features of the speech in each frame. The distribution $p_{\text {av }}(\mathcal{S}, \mathcal{V})$ is modeled by the GMM and is trained using a corpus of corresponding AV streams via the Expectation Maximization (EM) algorithm. 
Suppose that one of sources, say $s^{1}$, is a speech signal for which we have a video feature stream $\mathcal{V}^{1}$ extracted from the corresponding speaker's lip region. Then, the AVSS algorithm of $[12,14]$ tries to estimate the first row of demixing matrix $B^{1}$ for which the output $y^{1}=B^{1} \mathbf{x}$ produces spectral features $\mathcal{Y}^{1}$ as coherent as possible to the video features $\mathcal{V}^{1}$. This can be done by minimizing the AV incoherency score of the following AV model which is defined on each AV frame $k$ :

$$
\mathcal{M}_{\mathrm{GMM}}\left(\mathcal{Y}_{k}^{1}, \mathcal{V}_{k}^{1}\right)=-\log \left(p\left(\mathcal{Y}_{k}^{1}, \mathcal{V}_{k}^{1}\right)\right)
$$

However, due to the viseme-phoneme ambiguity problem $[28,29]$, it is possible that video features $\mathcal{V}^{1}$ in some frames be associated to many spectral configurations. Hence, the single frame criterion (5) will result in very poor separation. Consequently, they have proposed a batch-wise AV criterion which integrates joint $\log$-likelihood on all the $T$ frames of current batch $\tau$ :

$$
J_{\mathrm{av} G M M}\left(B ; \mathbf{x}_{\tau}, \mathcal{V}_{\tau}^{1}\right)=\sum_{k=1}^{T} \mathcal{M}_{\mathrm{GMM}}\left(\mathcal{Y}_{\tau}^{1}(k), \mathcal{V}_{\tau}^{1}(k)\right)
$$

The summation in (6) is based on the assumption that AV frames in consecutive frames are independent from each other.

In the rest of this text unless mentioned otherwise, we always consider a single row de-mixing vector denoted by $B$ corresponding to a single visual stream. For the sake of brevity we omit the superscript $(.)^{1}$ of variables. It is clear that in case of existence of multiple video streams corresponding to more than one speech sources, all the described methods can be repeated for each video stream.

\section{Audio-visual speech source separation using MLP AV modeling}

Here, a method is proposed for separation of the source of interest $s$ from $M$ mixed signals $\mathbf{x}$. The goal is to estimate $B$ such that $y=B \mathbf{x}$ be similar as possible to the original source $s . s$ is unknown but we have the visual stream $\mathcal{V}$ corresponding to it, we can estimate $B$ such that $\hat{\mathcal{V}}$ (the estimated visual stream corresponding to $y$ ), be as close as possible to $\mathcal{V}$.

A problem with objective function (6) of [12] and [14] is that it does not efficiently model the non-linear AV relation (as is discussed later in this section). Also it considers independence (i.i.d) assumption in modeling relation of consequent $\mathrm{AV}$ frames. We suggest to improve the AV criterion via more realistic assumptions.

Consider the batch-wise separation problem of equation (2) where every batch $\tau$ consists of $T$ frames. It is ideal to model and measure the degree of AV coherency on the joint whole sequences of audio $\mathcal{S}_{\tau}(1: T)$ and visual $\mathcal{V}_{\tau}(1: T)$ frames considering the true dependency among the variables. Let $\mathcal{M}_{\mathrm{IDL}}\left(\mathcal{S}_{\tau}, \mathcal{V}_{\tau}\right)$ be such an ideal model which measures the degree of incoherency between AV streams. Then, the de-mixing vector $B_{\tau}$ may be estimated by minimizing the ideal AV criterion $J_{\text {avIDL }}\left(B ; \mathbf{x}_{\tau}, \mathcal{V}_{\tau}\right)=$ $\mathcal{M}_{\text {IDL }}\left(\mathcal{Y}_{\tau}, \mathcal{V}_{\tau}\right)$.

However, training such an ideal model is not practical due to the need for large amount of AV training data and also due to its train and optimization complexity. Hence, considering some relaxation assumptions which factorizes the model to a combination of some reusable factor(s) is inevitable. The independent and identically distributed (i.i.d) assumption considered in GMM model of (6) is not a fit assumption for modeling the speech AV streams. Thus we propose an enhanced model with a weaker independence assumption. Instead of considering absolute independence between $\mathrm{AV}$ frames, we consider a conditional independence assumption that is the coherency of an AV frame can be estimated independent of other frames given a context of a few $(K)$ neighbor frames.

An extension of $p_{\text {av }}(\mathcal{S}, \mathcal{V})$ to model joint probability density function (PDF) of $K$ consecutive AV frames is not efficient. GMM and Gaussian distributions with full covariance matrices are not suitable for modeling large dimensional random vectors since the number of free parameters of these models is of order $O\left(d^{2}\right)$ relative to the dimension $d$ of input random vectors. Increasing the input dimension by concatenation of $K \mathrm{AV}$ frames will result in a very complex model with huge number of free parameters that are not used effectively.

We propose to use a MLP instead of GMM and mean square error (MSE) criterion instead of negative log probability (as incoherency measure) to provide an enhanced AV criterion. The number of free parameters of an MLP with narrow hidden layer(s) is of order $O\left(d_{i}+d_{o}\right)$ relative to dimensions $d_{i}$ and $d_{o}$ of its input and output. Moreover, MLP makes efficient use of its free parameters in learning non-linear AV relation, according to its hierarchical structure compared to shallow and wide structure of GMM. MLP, like GMM, is differentiable relative to its input. Hence, an objective function defined based on MLP can be optimized with fast convergence using derivative based algorithms.

\subsection{MLP audio visual model}

Having acoustic and visual streams of feature frames $\mathcal{S}$ and $\mathcal{V}$ extracted from pairs of corresponding AV signals $s$ and $\mathbf{V}$ (see Section 4.1), a context-dependent AV associator can be trained using a suitable non-linear function approximator: $\hat{\mathcal{V}}(k)=h\left(\mathcal{S}_{e}(k)\right)$, where $\mathcal{S} e(k)=E(\mathcal{S}(k-$ $K / 2-1: k+K / 2)$ ) is an embedded vector obtained from a context of $K$ audio frames around frame $k$. An option for embedding $E$, is to stacks the center frame of the context and the first-order temporal difference of other frames. In this paper, we adopt an MLP with $K$ input audio frames, one hidden layer of $N_{H}$ neurons and a single visual frame 
as output, to approximate the AV mapping $h($.). The MLPbased AV incoherency model $\mathcal{M}_{\mathrm{MLP}}$ is then defined as

$$
\mathcal{M}_{\mathrm{MLP}}(\mathcal{Y} e(k), \mathcal{V}(k))=\|\mathcal{V}(k)-h(\mathcal{Y} e(k))\|^{2}
$$

\subsection{Audio visual source separation algorithm}

To compensate for phoneme-viseme ambiguity, the MLP model must be used in a batch-wise manner. Hence, as in (6), AV criterion is boosted by integrating incoherency scores of $T$ frames in each batch $\tau$ :

$$
J_{\mathrm{avMLP}}\left(B ; \mathbf{x}_{\tau}, \mathcal{V}_{\tau}\right)=\sum_{k=1}^{T} \mathcal{M}_{\mathrm{MLP}}\left(\mathcal{Y} e_{\tau}(k), \mathcal{V}_{\tau}(k)\right)
$$

Beside the difference in negative log probability and mean square error, another difference between AV objective functions (6) and (8) is the form of independence assumption in measuring the incoherency. The former considers absolute independence (i.e., i.i.d.) between the frames while the later assumes conditional independence.

For each batch $\tau$ of mixed signals and having a visual stream $\mathcal{V}_{\tau}$ corresponding to one of the speech sources, the goal of separation is to find the de-mixing vector $B_{\tau}$. As in (3), this can be achieved by minimizing AV contrast function:

$$
B_{\tau}=\underset{B}{\operatorname{argmin}}\left\{J_{\mathrm{avMLP}}\left(B ; \mathbf{x}_{\tau}, \mathcal{V}_{\tau}\right)\right\}
$$

This can be done via first- or second-order derivativebased optimization methods. For example, using the delta rule of gradient decent, we have

$$
B_{\tau}(i)=B_{\tau}(i-1)-\eta \frac{\partial J_{\mathrm{avMLP}}\left(B ; \mathbf{x}_{\tau}, \mathcal{V}_{\tau}\right)}{\partial B}
$$

where $\eta$ is the learning rate which either is set to a fixed small number or is adjusted using line search. The gradient of $J_{\text {av MLP }}$ with respect to $B$ (omitting constant parameters for brevity) is calculated as:

$$
\begin{aligned}
\frac{\partial J_{\mathrm{av} \mathrm{MLP}}(B)}{\partial B} & =\sum_{k=1}^{T} \frac{\partial \mathcal{M}_{\mathrm{MLP}}\left(\mathcal{Y}_{e_{\tau}}(k)\right)}{\partial B} \\
& =\sum_{k=1}^{T} \frac{\partial \mathcal{M}_{\mathrm{MLP}}\left(\mathcal{Y}_{e_{\tau}}(k)\right)}{\partial \mathcal{Y}_{e_{\tau}}(k)} \frac{\partial \mathcal{Y}_{e_{\tau}}(k)}{\partial B}
\end{aligned}
$$

In the last summation, the first term is gradient of MLP AV model with respect to its input acoustic context $\mathcal{Y}_{e}(k)$ and the second term is gradient of acoustic features with respect to the de-mixing model $B$. Gradient-based algorithm iteratively minimizes the problem (9). Starting from an initial point $B_{\tau}(0)$, at each iteration $i$, the gradient (11) is calculated, and using (10) or a quasi-Newton method, the improved de-mixing vector $B_{\tau}(i+1)$ is estimated. This continues until the change in the norm of $B_{\tau}$ or $J_{\mathrm{av} \operatorname{MLP}}\left(B_{\tau}\right)$ becomes smaller than a pre-defined threshold.

Since the AV contrast function is not convex, the optimization algorithm is prune to local minima. Thus, selection of a good initialization point $B_{\tau}(0)$ is important. A simple option may be to start from random initial points multiple times. Most ICA algorithms (including FastICA [26] and JADE [27]) start from uncorrelated or white signals. Thus, another suggestion for initial point $B_{\tau}(0)$ is to apply PCA on mixed signals $\mathbf{x}_{\tau}$ of the current batch $\tau$ and, among eigenvectors, select a vector $W$ that produces a signal $y=W \mathbf{x}$ which is most coherent with the visual stream $\mathcal{V}_{\tau}$ and use it as the initial point $B_{\tau}(0)$.

Both the proposed and existing AVSS algorithms do not suffer from the permutation ambiguity due to the informed nature of AV contrast functions. Nevertheless, the scale indeterminacy should be considered in design of AV contrast function and optimization method. AV model must be invariant regarding a constant gain to audio signal; that is, it must comply with the following constraint:

$$
J_{\mathrm{av} \operatorname{MLP}}(\alpha B ; \mathbf{x}, \mathcal{V})=J_{\mathrm{av} \operatorname{MLP}}(B ; \mathbf{x}, \mathcal{V})
$$

\subsection{AVSS using AV coherency and independence criterion} Although the existing and the proposed AV coherencybased methods provide improvements in speech source separation, but these methods totally neglect the useful constraint of independence of the sources. The statistical independence criteria used by ICA methods has been successful in many BSS methods. In this section, we consider the benefit of using AV coherency and statistical independence together to gain more enhancement in speech source separation.

\subsubsection{Video-selected independent component}

Due to permutation indeterminacy (4), separated signals from ICA methods can not directly be used in real speech processing applications. Further, to calculate output signal to interference ratio (SIR) performance of ICA methods, it is required to know which of the de-mixed signals is related to the source of interest.

AV incoherency scores from AV models may be incorporated to introduce loosely coupled video-assisted ICA [14]. For that, in each batch of signals, sources are estimated by ICA method, and the source with minimum incoherency relative to the visual stream is selected as speech of interest. JADE [27] is one of the most successful ICA methods because of its accurate separation and its uniform performance (equivariance property). In this paper, we use JADE algorithm together with MLP audiovisual model (for relevant source selection) as the video assisted JADE (denoted by JADE-AV). 


\subsubsection{Hybrid video coherent and independent component analysis}

Contrary to the previous section where a sequential and loose combination of ICA and AV coherency model was considered, here we propose a parallel and tight combination using a hybrid criterion which benefits from normalized kurtosis as a statistical independence measure in conjunction with the AV coherency measure.

Kurtosis and neg-entropy are used in ICA methods such as FastICA [26] which work by maximizing the nonGaussianity. The first kurtosis-based BSS method was presented in [30] to separate sources via deflation. It starts by pre-whitening the observed signals. Then the first source is estimated as $y=B \mathbf{x}^{\prime}$ from white observations $\mathbf{x}^{\prime}$ using a normalized de-mixing vector $B$. It is estimated by maximizing the kurtosis of $y$, defined as $\operatorname{kurt}(y)=$ $E\left\{y^{4}\right\}-3\left(E\left\{y^{2}\right\}\right)^{2}$ (for zero-mean $y$ ) that is done via a gradient-like method. The kurtosis value is zero for Gaussian signals while it is positive or negative for signals with super- or sub-Gaussian distributions. If both super- and sub-Gaussian sources are expected to be extracted, then absolute or squared value of kurtosis must be maximized.

In [26], Hyvarinen et al. proposed a fast fixed point algorithm for solving the constrained optimization of the kurtosis and a family of other neg-entropy-based criteria under the normalized constraint for $B$ which resulted in the well-known FastICA algorithm.

The reason for pre-whitening and forcing normalized constraint on $B$ is that the kurtosis is not scale invariant (i.e. $\left.\operatorname{kurt}(\alpha y)=\alpha^{4} \operatorname{kurt}(y)\right)$ and hence it depends both on energy and non-gaussianity of the signal. In [31] and [32] normalized kurtosis defined as

$$
\operatorname{kurt}_{n}(y)=\frac{\operatorname{kurt}(y)}{\left(E\left\{y^{2}\right\}\right)^{2}}
$$

is adopted on direct observations. The normalized kurtosis is scale invariant (i.e. $\operatorname{kurt}_{n}(\alpha y)=\operatorname{kurt}_{n}(y), \forall \alpha \neq 0$ ). Hence, it eliminates the necessity for pre-whitening and normalization constraint on the de-mixing vector $B$. To gain further improvement, we propose a hybrid criterion based on combination of the AV criterion (8) and the normalized kurtosis:

$$
J_{\mathrm{avICA}}\left(B ; \mathbf{x}_{\tau}, \mathcal{V}_{\tau}\right)=J_{\mathrm{avMLP}}\left(B ; \mathbf{x}_{\tau}, \mathcal{V}_{\tau}\right)-\lambda \operatorname{kurt}_{n}\left(B \mathbf{x}_{\tau}\right)
$$

where $\lambda$ is a positive regularization coefficient. Since speech signal is known to have super-Gaussian distribution $[33,34]$, the kurtosis term is added with negative sign such that it tends to be maximized during minimization of (14).

It must be noted that, in short time durations, the kurtosis score is not robust and does not provide signif- icant improvement. Thus, (14) is developed to be used for convolutive case where quite large batches are considered. In fact, our tests revealed that for small batch sizes used in instantaneous mixtures, the performance of the AV method using kurtosis penalty does not improve compared to the pure AV method.

\subsection{Toward a time domain AVSS for convolutive mixtures} Here, we consider convolutive mixtures defined by a MIMO system of $M \times N$ FIR filters $\mathbf{A}=\left[A_{i j}\right]$. The mixture system can be represented in the the Z-domain as

$$
\mathbf{X}(z)=\mathbf{A}(z) \mathbf{S}(z)
$$

We are interested in estimation of a $1 \times M$ row vector $B(z)$ of de-mixing FIR filters which separates the source $S^{1}(z)=B(z) \mathbf{X}(z)$ that is as coherent as possible with the video stream $\mathbf{V}^{1}$. In [31], a time domain algorithm based on maximizing (normalized) kurtosis is presented which deflates sources one-by-one using non-causal two-sided FIR filters. We consider it as our baseline audio-only convolutive method in our experiments. Following [35], we define an embedded matrix notation which transforms the convoltive mixture (15) to an equivalent instantaneous mixture. Let $\mathbf{x}^{\prime}(n)$ be an embedded column vector defined in each time step $n$ as:

$$
\begin{aligned}
\mathbf{x}^{\prime}(n)= & {\left[x^{1}(n+L), \cdots, x^{1}(n-L), \cdots, x^{M}(n+L), \cdots,\right.} \\
& \left.x^{M}(n-L)\right]^{T}
\end{aligned}
$$

It contains $M(2 L+1)$ observation samples and using it the convolutive de-mixing process for separation of signal $s^{1}$ can be expressed as $y(n)=B \mathbf{x}^{\prime}(n)$ where $B$ is a row vector containing coefficients of $M$ de-mixing FIR filters each one having $2 L+1$ taps. This is just an instantaneous mixture with $M(2 L+1)$ virtual (embedded) observations and can be solved using the kurtosis-based method of [31] or using our proposed criteria (14).

As a final note, it should be mentioned that the reference method of [31], can estimate de-mixing filters up to a scale and time delay. Thus, a cross-correlation step is necessary to fix the possible delay of filters. For further details please refer to [31]. When dealing with convolutive mixtures, it is necessary to calculate the objective scores on longer segments of signals since there are larger number of parameters to estimate.

\section{Audio-visual data and models}

Audio-visual corpus and model are building material toward realization and evaluation of the proposed AVSS algorithm which is a data-driven method. In the following, we look at AV corpus creation and models training. 


\subsection{Audio-visual data}

To evaluate the proposed algorithm, we have recorded a proper AV corpora which is comparable in (size and complexity) to the corpora used in former research. Unlike $[11,12]$, we have not used lip blue make-ups in data recordings since we do not need lip segmentation for extraction of geometric features such as width and height. Instead, the pixel gray values of speaker's mouth region are used to extract the visual parameters. We have recorded two different types of corpora. The first corpus consists of discrete Persian and English alphabet and digits with a vocabulary size of 78 words $(32+10$ Persian and $26+$ 10 English alpha-digits). The second corpus is continuous and consists of 140 verses of Persian poets. Both corpora are uttered by a male speaker. Each corpus is recorded two times. The first recording is used for training AV models and the second recording is used in evaluation phase.

In each recording, camera is focused on the speaker's mouth and a video stream together with a mono audio stream is recorded. The raw video is captured in VGA size, true-color format (RGB 24 bits/pixel 8 bits/color) and at the frame rate of $F_{r} \simeq 30 \mathrm{fps}$ and audio is recorded using $16 \mathrm{bits} / \mathrm{sample}$ and at sampling frequency of $F_{s}=$ $16,000 \mathrm{~Hz}$. The final mouth region video used in the experiments of this paper, is stored in true-color $160 \times$ 120 resolution frames. Sample lip region images from audio-visual corpus are shown in Figure 1a.

\subsection{Audio and video parameter extraction}

As discussed before, speech signal and lip image frames are high-dimensional data with sparse information related to our task. Thus, parametrizing audio and visual frames to compact vectors is necessary. Here, we clarify the methods for audio and visual feature extraction.

\subsubsection{Audio parametrization}

In most speech processing tasks, log spectral envelope (cepstral) features are utilized as effective features. We use PCA projected (whitened) log power spectral density for the speech frames parametrization. Let $\mathcal{Y}(k)=$ $f(y(k))=f(B \mathbf{x}(k))$ be acoustic feature mapping function which extracts $k_{a}$ spectral envelope features $\mathcal{Y}$ from every frame $k$ of the estimated signal $y$. In practice, audio features $\mathcal{Y}$ are extracted from the spectrum $Y$ of the demixed signal $y$. Since for the separation algorithm we need to efficiently calculate $\mathcal{Y}$ and its derivative with respect to the de-mixing vector $B$, we define an alternate audio feature extractor function $F($.) which efficiently extracts features from the frequency domain representation of $y$ :

$$
\mathcal{Y}(k)=F(Y(k))=F(B \mathbf{X}(k))
$$

where $Y=\mathscr{F}\{y\}$ and $\mathbf{X}=\mathscr{F}\{\mathbf{x}\}$ are the fast Fourier transform (FFT) of $y$ and $\mathbf{x}$, respectively. In the right-hand side (RHS) of (17), we have used the linear property of FFT that is, for every matrix $\mathbf{B}, \mathscr{F}\{\mathbf{B x}\}=\mathbf{B} \mathscr{F}\{\mathbf{x}\}$. Thus, we can pre-calculate $\mathbf{X}$ using FFT and then for any value of the de-mixing vector $B$ the frequency domain de-mixed signal $Y$ (and its derivative) can be efficiently obtained without FFT recalculation. As in [14], we have considered $n=32$ spectral coefficients in the range $[0,5,000] H z$ as $Y(k)$ for each frame. Power spectrum vector of each frame $Y(k)$

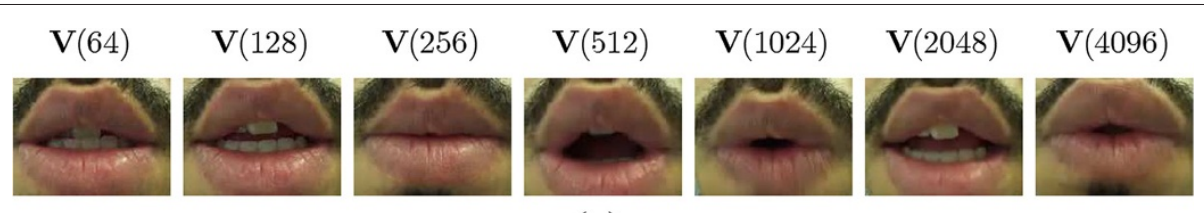

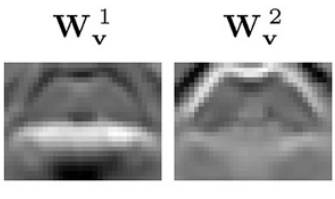
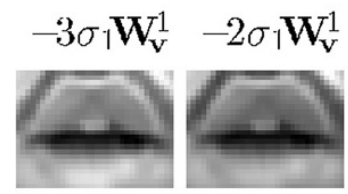
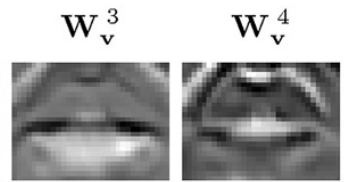

(b)
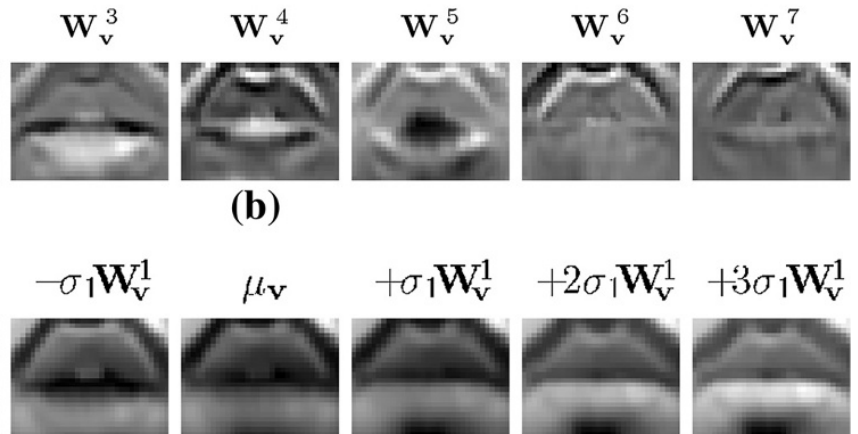
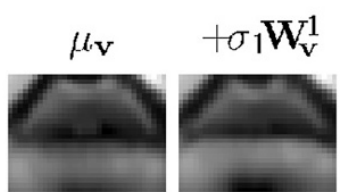
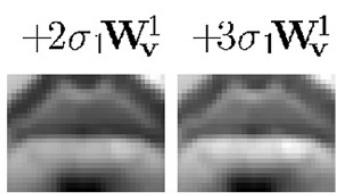

(c)

Figure 1 Visual modality: data and parametrization. (a) Shows some frames ( $k=64,128,256,512,1024,2048$ and 4096) of visual input $\mathbf{V}(k)$ from train set of the poet verses corpus. (b) Shows top seven eigen-lips $\mathbf{W}_{v}^{i}$ with largest eigenvalues. (c) Demonstrates the effect of varying the average image of all frames in the direction of the major principal axis $\left(\mathbf{W}_{v}^{1}\right)$ with $-3,-2,-1,0,1,2$ and 3 times of square root of the corresponding eigenvalue $\left(\sigma_{1}\right)$ which has resulted in synthetic opening and closing of lips. 
is then defined as $P S_{Y}(k)=\left[Y(k) \otimes Y^{*}(k)\right]^{T}$, where $\otimes$, $(.)^{*}$ and $(.)^{T}$ are element-wise product, complex conjugation and transpose operators. Although the lip and the speech spectral envelope shapes are correlated, but there is not any meaningful relation between the lip shape and speech loudness (energy). Thus, it is important to normalize the energy of power spectrum in each frame resulting in power spectral density (PSD). The PSD coefficients are then converted to decibels $(\mathrm{dB})$ using logarithm

$$
\log \operatorname{PSD}_{Y}(k)=\log \left(\frac{P S_{Y}(k)}{\|Y(k)\|^{2}}\right)
$$

Finally, whitening is applied to reduce the dimension of acoustic feature vectors to $k_{a}$ elements. The acoustic whitening matrix $\mathbf{W}_{a} \in \mathbb{R}^{n \times k_{a}}$ is computed from the train data using eigen-value decomposition and is used to project train and test feature vectors to $k_{a}$-element compact spectral acoustic vectors. The overall acoustic feature extraction function $F(Y)$ is defined as follows:

$$
\mathcal{Y}(k)=F(B ; \mathbf{X}(k))=\mathbf{W}_{a}^{T} \log \left(\frac{\left[(B \mathbf{X}(k)) \otimes(B \mathbf{X}(k))^{*}\right]^{T}}{\|B \mathbf{X}(k)\|^{2}}\right)
$$

The Jacobian of $F$ with respect to $B$ is derived in the Appendix in Equations 22, 23 and 24. The derived formulas are efficient and do not need FFT recalculation for different values of $B$. It is also worth to mention that the mapping $F$ is invariant regarding a scalar multiplication (i.e. $F(\alpha B ; \mathbf{X}(k))=F(B ; \mathbf{X}(k)), \forall \alpha \neq 0)$. A property that entails gain invariance property (12) in AV contrast functions (6) and (8).

\subsubsection{Video parametrization}

In previous works, such as $[11,12,14]$, authors have used geometric lip parameters that need lip contour detection to estimate the width and height of interior lip contour. We extract holistic visual features from all pixels of the mouth region. This requires less computation and does not require contour fitting. Let function $g($.) be visual feature mapping function which extracts $k_{v}$ visual features from any video frame. We assume that mouth region can be extracted from video using detection and tracking algorithms. There exists efficient parametric head tracking algorithms such as [36] which can be adopted for this task. The corpus used in this paper simply provides lip region in each frame. To extract $k_{v}$ visual features, the mouth region of each frame is shrunk to $32 \times 24$ pixels and then reshaped to $768 \times 1$ image vectors. Finally, a PCA transform is applied to extract visual features. The PCA matrix $\mathbf{W}_{v} \in \mathbb{R}^{768 \times k_{v}}$ is computed from the train data and is used to project train and test mouth region images to $k_{v}$-element visual parameter vectors. Figure $1 \mathrm{~b}$ represents top major eigen-vectors (eigen-lips) in order.

The overall visual feature extraction function is defined as normalized projected gray values of mouth region: $\mathcal{V}(k)=g(\mathbf{V}(k))=\mathbf{Q}_{v}^{T} \mathbf{W}_{v}^{T} \mathbf{V}(k)$, where $\mathbf{Q}_{v}$ is the diagonal scaling matrix calculated from square root of corresponding eigen-values.

To assess and understand the virtue of visual features, a simple yet insightful simulation is illustrated in Figure 1c. In PCA, the eigenvector with largest eigenvalue captures most of the variance of dataset. Most variance of lip images during speaking is along opening and closing of lips. Thus, it is expected that the principal eigenvector $\mathbf{W}_{v}^{1}$ will model this direction of variation. To check this, we calculated mean vector $\mu_{v}$ of all video frames in poetverses train corpus and illustrated its variations along the principal eigenvector $\mathbf{W}_{v}^{1}$ with negative and positive integer multiplies of square root of corresponding eigenvalue $\sigma_{1}$. Results presented in Figure 1c show that this has resulted in synthesized opening and closing of lip images.

Figure 2a,c demonstrates two segments of discrete and continuous speech from alpha-digits and poet-verses corpora and Figure $2 b, d$ shows the corresponding first visual feature (before normalization). In discrete or slow speech, first visual feature shows a quasi-periodic shape corresponding to the periodic lip opening and closing. In continuous or fast speech, lip opening and closing is partial and this makes it more complex.

\subsection{Building audio-visual models}

In addition to estimation of transforms $\mathbf{W}_{a}$ and $\mathbf{W}_{v}$, that are part of the AV feature mapping functions $f($.$) and g($.$) ,$ the train set of each corpus is used to learn the AV models. The training set consists of synchronous sequences of AV pairs $(\mathcal{S}(k), \mathcal{V}(k))$ extracted from the raw AV data. For training models with $K>1$, first, the embedded acoustic stream $\mathcal{S}_{e}$ is formed by $K$-fold embedding of frames of acoustic stream $\mathcal{S}$. Instead of stacking the $K$ frames of context, it is better to stack the center frame together with temporal difference of other frames. This reduces the redundancy in the embedded vector. Then, embedded pairs $\left(\mathcal{S}_{e}(k), \mathcal{V}(k)\right)$ are used to train models. Both GMM and MLP models are trained with different context sizes for fair comparison. But as experimental results of Section 5.1 shows, GMM degrades with $K>1$.

For training GMM models, AV components of each pair are concatenated and considered as samples of joint PDF $p_{\mathrm{av}}(.$, .). These samples are used for estimation of GMM parameters using maximum likelihood via expectation maximization (EM) algorithm [37]. GMM distributions with various configuration of parameters $\left(k_{a}, k_{v}, N_{M}, K\right)$ are trained. To assure good training, for each setting, 


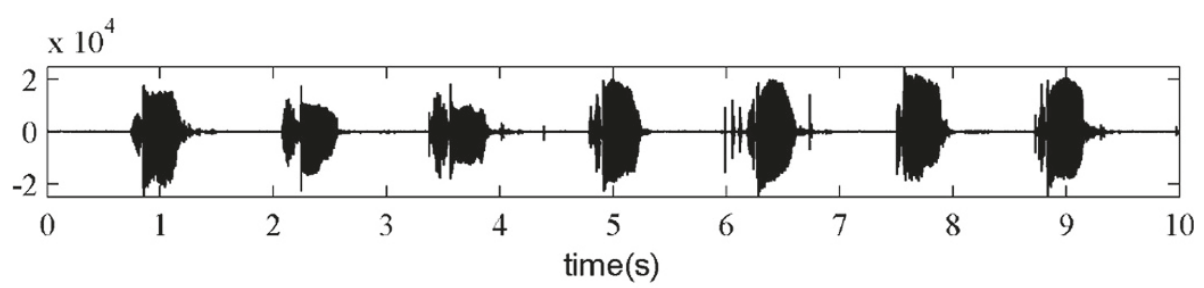

(a)

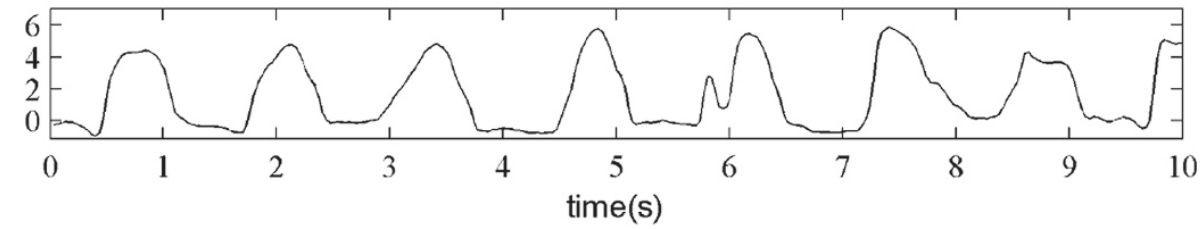

(b)

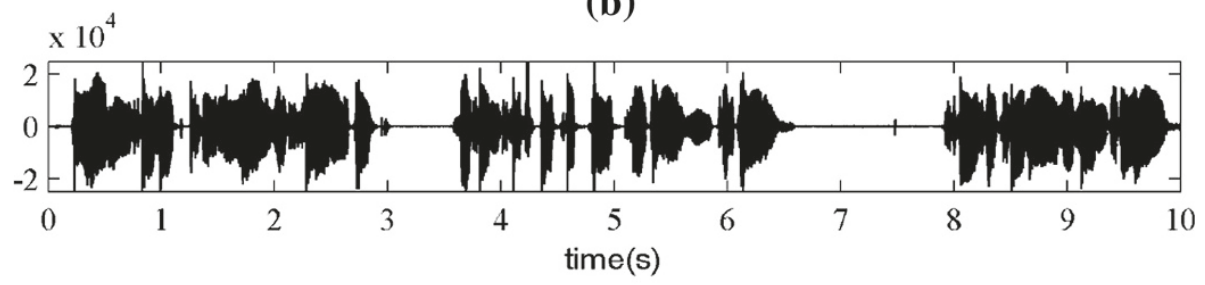

(c)

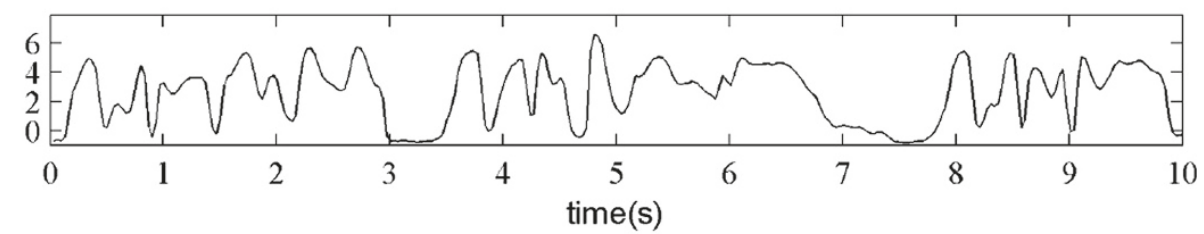

(d)

Figure 2 Sample segments of speech signal and first visual feature corresponding to them. $(\mathbf{a}, \mathbf{c})$ discrete and continuous speech segments from alpha-digits and poet-verses corpora. (b,d) First visual feature corresponding to $(a, c)$.

GMM distribution is trained 20 times using EM with random initialization and the best model is selected based on a validation subset of training data. Regularization by adding a small positive number in range $\left[10^{-10}, 10^{-2}\right]$ to diagonal elements of covariance matrices was adopted to hold positive definiteness where necessary (specially for models with larger random vector dimensions).

MLP AV models are also trained on AV pairs $\left(\mathcal{S}_{e}(k), \mathcal{V}(k)\right)$ with $\mathcal{S}_{e}(k)$ as input and $\mathcal{V}(k)$ as output. MSE criterion between true and estimated outputs $\mathcal{V}(k)$ and $\hat{\mathcal{V}}(k)$ is used as the performance measure in training. This is the same criterion as what is used in contrast function (8). Networks were trained using the LevenbergMarquardt algorithm [38] and via early stopping based on validation subset to avoid over-fit. As for GMM, MLP models with various configuration of parameters $\left(k_{a}, k_{v}\right.$, $\left.N_{H}, K\right)$ are trained. To avoid local minima in training, each model is trained 20 times with random initialization and the best model is selected based on the validation subset.

\section{Experiments and results}

For evaluation of the proposed method, we have conducted four sets of experiments at different stages. First fitness of AV models in capturing AV coherency is evaluated with some initial experiments providing enough data for hyper-parameter selection of models. Then, multiple source separation experiments on regular $(N \times N)$ and degenerate $(M \times N, M<N)$ cases are conducted to compare performance of proposed MLP-based AVSS method with GMM-based AVSS and JADE-AV method (defined in Section 3.3.1). Finally, experiments on convolutive $2 \times 2$ mixtures with filters of different length are presented to compare performance of the audio-only and the proposed hybrid method.

\subsection{Audio-visual models assessment and selection}

In this experiment, we pre-evaluate fitness of AV models and explore the effect of different parameters on their performance. Both MLP- and GMM-based AV models need 
training and have hyper-parameters to be selected. We should choose proper dimensions $k_{a}$ and $k_{v}$ of acoustic and visual parameters, the embedding context size $K$, the number of hidden neurons $N_{H}$ of MLP and the number of Gaussian components $N_{M}$ of GMM models. Although validation scores of trained models can be used to select best GMM and MLP models, but selection of models based on their capability of discrimination between coherent and incoherent speech is more reasonable since models are aimed to be used for source separation. Furthermore, such an experiment provides insights in virtual potentials of coherency-based AVSS methods.

\subsubsection{Audio-visual pure relevant source detection}

In this experiment, we compare incoherency scores between a visual stream $\mathbf{V}^{1}$ and two pure audio signals: a coherent signal $s^{1}$ and an irrelevant signal $s^{2}$. For each frame in the test set, the signal which produces minimum incoherency score is recognized to be coherent with $\mathbf{V}^{1}$. Experiments are performed for both AV models $\mathcal{M}_{\mathrm{GMM}}(\mathcal{S}, \mathcal{V})(5)$ and $\mathcal{M}_{\mathrm{MLP}}(\mathcal{S}, \mathcal{V})(7)$. For each model, different values of hyper-parameters $k_{a} \in\{2,4,6,8,10,12\}$, $k_{v} \in\{2,4,6,8\}, N_{M}, N_{H} \in\{4,8,12,16,20,24,28\}, K \in$
$\{1,2,3,4,5,6\}$ are examined. Finally, the percent of all frames which signal $s^{1}$ is truly selected is reported as classification accuracy for different values of batch size $T$.

In [14], authors have evaluated the classification rate just against a single irrelevant signal which is uttered by a different male speaker. Our initial experiments revealed that classification accuracy for different irrelevant signals is variable depending on the speaker, the speech content of signal and alignment of silent parts of coherent and incoherent signals. Thus, to provide classification rates with high confidence, we conducted multiple simulations by performing coherency classification on the relevant signal $s^{1} y$ against six distinct speech signals for $s^{2}$ and reported the average recognition rate as the performance of models.

Furthermore, it is possible that AV models, in addition to AV coherency of speech, capture some parts of AV identity of speaker. To check for this, we chose coherent and irrelevant speech signals both from the same speaker. As much as AV models have captured speaker identity, this provides a classification problem which is more confusing and complex for them relative to choosing irrelevant signals from different speaker(s).

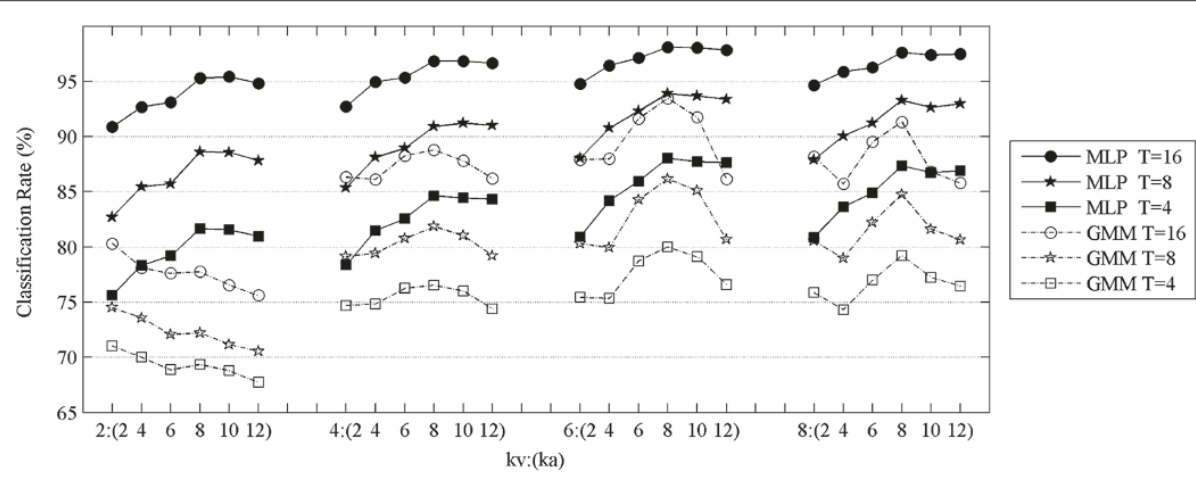

(a)

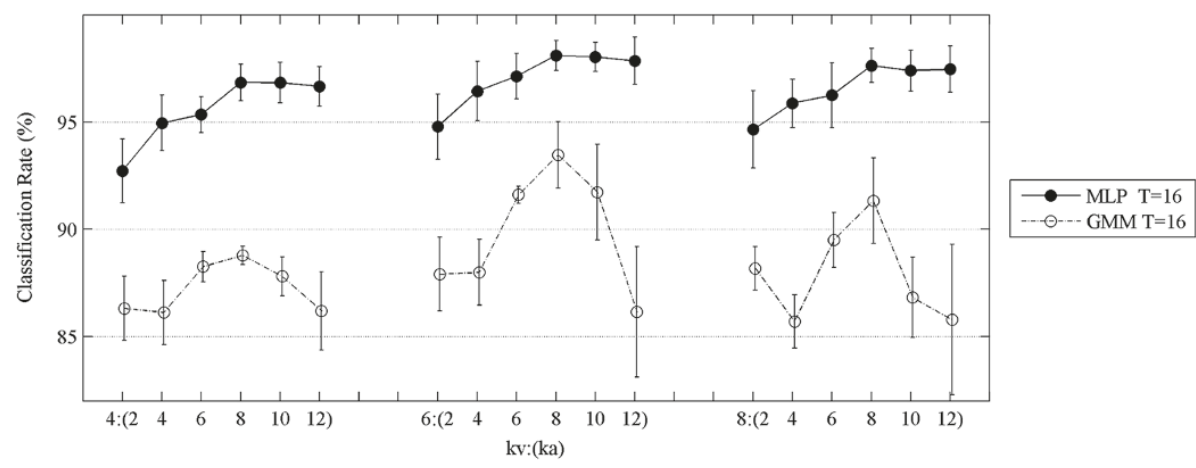

(b)

Figure 3 Video coherent speech detection on pure relevant and irrelevant signals. (a) Results for different configurations of model type (MLP,GMM), $k a(2,4,6,8,10,12), k v(2,4,6,8)$ and $T(4,8,16)$. (b) Error bars (95\% confidence interval) for curves with $T=16$. Other free parameters are marginalized by maximum selection (see text for more details). 
Table 1 Optimal embedding context size $(K)$ and model sizes ( $N_{M}$ of GMM and $N_{H}$ of MLP)

\begin{tabular}{|c|c|c|c|c|c|c|c|c|}
\hline \multicolumn{5}{|c|}{$\operatorname{GMM}\left(K / N_{M}\right)$} & \multicolumn{4}{|c|}{$\operatorname{MLP}\left(K / N_{H}\right)$} \\
\hline$k_{a}$ & $k_{v}: 2$ & 4 & 6 & 8 & 2 & 4 & 6 & 8 \\
\hline 2 & $1 / 4$ & $1 / 16$ & $1 / 28$ & $1 / 8$ & $4 / 20$ & $4 / 16$ & $4 / 20$ & $6 / 28$ \\
\hline 4 & $1 / 24$ & $1 / 24$ & $1 / 24$ & $1 / 20$ & $4 / 20$ & $3 / 16$ & $2 / 24$ & $3 / 16$ \\
\hline 6 & $1 / 28$ & $1 / 20$ & $1 / 24$ & $1 / 24$ & $4 / 8$ & $2 / 24$ & $3 / 16$ & $2 / 8$ \\
\hline 8 & $1 / 8$ & $1 / 8$ & $1 / 28$ & $1 / 28$ & $2 / 20$ & $2 / 16$ & $2 / 28$ & $2 / 16$ \\
\hline 10 & $1 / 24$ & $1 / 28$ & $1 / 28$ & $1 / 24$ & $2 / 16$ & $2 / 28$ & $2 / 16$ & $2 / 16$ \\
\hline 12 & $1 / 8$ & $1 / 16$ & $1 / 20$ & $1 / 20$ & $4 / 4$ & $2 / 16$ & $2 / 20$ & $4 / 8$ \\
\hline
\end{tabular}

Optimal values are selected using cross validation for different values of $k_{a}$ and $k_{v}$.

Comprehensive classification rates are presented in Figure 3 for both GMM and MLP models and for different values of $k_{a}, k_{v}$ and $T$ as free parameters. In this figure, other parameters $\left(N_{H}\right.$ of MLP, $N_{M}$ of GMM and $K$ of both models) are marginalized by selecting the maximum accuracy among them.

Table 1 presents optimal values for hidden (marginalized) parameters $\left(N_{H}, N_{M}\right.$ and $K$ ) of Figure 3 for different $k_{a}$ and $k_{\nu}$ configurations in both GMM and MLP models.

The common trends in classification rates of Figure 3 reveals following points:

1. Accuracy of both MLP and GMM models is enhanced by increasing the number of batch frames $T$, acoustic features $k_{a}$ and visual features $k_{v}$.

2. Among these factors, batch size $T$ has the highest impact and this is followed by $k_{a}$; finally, $k_{v}$ has the lowest impact.

Comparing the trends in Figure 3 for MLP and GMM models, also reveals interesting points:

1. MLP model performs significantly better relative to GMM in various AV dimensions.
2. In lower visual dimensions $\left(k_{v}=2\right)$, MLP outperforms with a $10-15 \%$ gap relative to GMM model. In this case, even performance of MLP with worst condition (batch size $T=4$ ) is $5-6 \%$ higher than GMM with best condition (batch size $T=16$ ).

3. In higher visual dimensions $\left(k_{v}=6,8\right)$, the difference between GMM and MLP is somewhat reduced.

4. The improvements by increasing number of features $k_{a}$ and $k_{v}$ is bounded. For $k_{v}>8$, in MLP, $k_{v}>6$ in GMM and $k_{a}>8$ in both models, no more significant enhancement is achieved. The model complexity increases in $O\left(K \cdot k_{a}+k_{v}\right)$ for MLP and $O\left(\left(K . k_{a}+k_{v}\right)^{2}\right)$ for GMM and in some point, this results in over-complex models for the problem (considering the amount of available training data).

5. Contrarily, improvements by increasing batch size $(T)$ continues upward and may reach perfect accuracy for enough large $T$ values. This is because the value of $T$ does not change the model size while increasing it introduces more information for decision making. However, it is important to mention that for real AVSS tasks, we cannot increase $T$ arbitrarily. This makes the stationary assumption considered in the mixture model (2) invalid. Hence, there is a trade-off on the value of $T$ between the AV model accuracy and the mixing model fitness.

Finally, results of optimal $K, N_{H}$ and $N_{M}$ values presented in Table 1 reveals that

1. In various $k_{a}$ and $k_{\nu} \mathrm{s}$, GMM always has performed better with $K=1$ frames in embedded context which means GMM can not capture temporal dynamics by frame embedding due to quadratic order of parameters.

2. MLP always has performed better with $K=2$ frames (for greater $k_{a}$ ) or $K=4,6$ frames (for smaller $k_{a}$ ) in embedded context showing that it can capture some temporal dynamics.

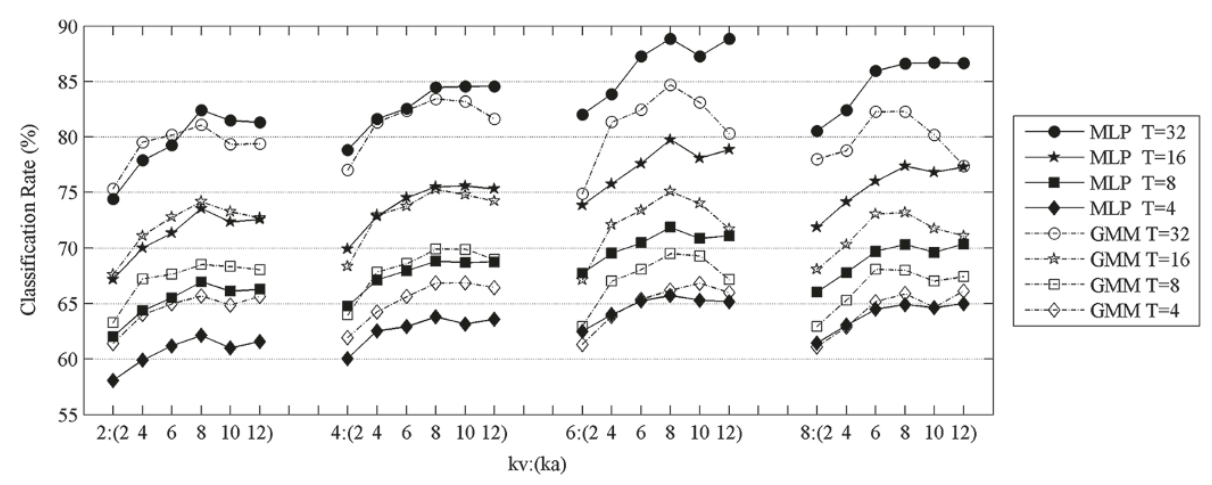

Figure 4 Video coherent speech detection on mixed relevant and irrelevant signals. Both relevant and irrelevant signals are mixed at different but close SIR levels. Other configurations are the same as in Figure 3 (see text for more details). 
Table 2 Average input (mixed) SIRs in decibels for both corpora and for different $\boldsymbol{M} \times \mathbf{N}$ mixing matrices

\begin{tabular}{|c|c|c|c|c|c|c|c|c|}
\hline \multirow{2}{*}{ Ch. $i$} & \multicolumn{4}{|c|}{ Alpha digits } & \multicolumn{4}{|c|}{ Poet verses } \\
\hline & $2 \times 2$ & $2 \times 3$ & $3 \times 3$ & $5 \times 5$ & $2 \times 2$ & $2 \times 3$ & $3 \times 3$ & $5 \times 5$ \\
\hline Ch. 1 & 0.6 & -2.5 & -3.2 & -9.0 & -0.7 & -3.9 & -4.5 & -10.2 \\
\hline Ch. 2 & -1.8 & -4.8 & -5.9 & -8.6 & -3.2 & -6.1 & -7.3 & -9.9 \\
\hline Ch. 3 & - & - & 0.4 & -8.8 & - & - & -0.9 & -10.1 \\
\hline Ch. 4 & - & - & - & -8.2 & - & - & - & -9.4 \\
\hline Ch. 5 & - & - & - & -6.7 & - & - & - & -8.0 \\
\hline
\end{tabular}

SIRs values are calculated with respect to $s^{1}\left(S \operatorname{IR}\left(x^{i} \mid s^{1}\right)\right)$ and they are averaged over all simulated input random matrices and all frames for each corpus.

3. Both GMM and MLP models exploit maximum average number of latent units in $k_{v}=6$ which seems to be efficient optimal visual dimension size according to results of Figure 3.

\subsubsection{Audio-visual mixed relevant source detection}

Recall that classification results in Section 5.1.1 are based on comparing the incoherency scores between pure relevant and irrelevant speech signals. This entails that AV models are well suited for selection of a clean relevant source among multiple available irrelevant signals. For example, it will perform well for relevant source selection in AV-assisted ICA-based source separation method (i.e. JADE-AV) discussed in Section 3.3.1.

In AV separation algorithms, models must provide scores for signal of relevant source which is more or less contaminated by other sources specially during first iterations of the optimization algorithm. Hence, a good AV model must be such that it provides decreasing incoherency scores for increasing amounts of SIR. Therefore, we conducted another experiment to assess how well AV models comply with this property. Let $\xi_{i}=s^{1}+\alpha_{i} s^{2}$ be a mixed signal composed of source $s^{1}$ coherent with visual stream $\mathbf{V}^{1}$ and an irrelevant speech or acoustic signal $s^{2}$. Mixed signals at different SIRs can be generated using different values for mixing coefficient $\alpha_{i}$. We generated a set of mixed signals $\xi_{i}$ with SIRs in range $[-5,30] \mathrm{dB}$ and performed classification using incoherency score comparisons between signal pairs $\left(\xi_{i}, \xi_{i+1}\right)$ at different SIR levels. Here, the classification accuracy is defined as percent of all frames which the signal with higher SIR is selected.
Figure 4 shows average classification accuracy measured at different SIR levels for all GMM and MLP models tested in previous experiment.

Generally, trends of Figure 4 shows similar properties as was discussed for Figure 3. The major point is that classification accuracies of best models on mixed signals $\xi_{i}, \xi_{i+1}$ is something about $10 \%$ less relative to classification of pure relevant and irrelevant signals. Such a degradation is predictable since signals $\xi_{i}$ and $\xi_{i+1}$ are very similar. But the interesting note is that superior models in the pure classification have approximately kept their superiority in the mixed case. This means that optimal model configurations which are better for classification task, may keep their position in separation task. As before MLP models are superior to GMM models but the large gap between them is somewhat reduced.

\subsection{Source separation experiments}

\subsubsection{Separation performance criterion}

In our experiments, we will simulate the mixing process using some mixing matrices. Thus, we have original source signals and it is possible to calculate the SIR of each acoustic source specially the source of interest $\left(s^{1}\right)$ in all mixed and de-mixed signals. Let $e\left(s^{i}\right)$ be the energy of source $i$ and $x^{i}$ be the $i^{t h}$ mixed signal produced by the mixing matrix $A$. Then SIR of $s^{1}$ in each of input-mixed observations can be calculated as

$$
\operatorname{SIR}\left(x^{i} \mid s^{1}\right)=10 \log _{10}\left(a_{i 1}^{2} e\left(s^{1}\right) / \sum_{j=2}^{N} a_{i j}^{2} e\left(s^{j}\right)\right)
$$

where $a_{i j}$ is the element of mixing matrix at positions $i, j$. The input SIR is useful for analysis of complexity of mixing matrices utilized in simulations. Similarly, consider $B$ as estimated de-mixing vector for source $s^{1}$ and let $G=B A$ be the global mixing and de-mixing vector for this source. Then output SIR of $s^{1}$ in estimated de-mixed signal $y$ can be calculated as:

$$
\operatorname{SIR}\left(y \mid s^{1}\right)=10 \log _{10}\left(g_{11}^{2} e\left(s^{1}\right) / \sum_{j=2}^{N} g_{1 j}^{2} e\left(s^{j}\right)\right)
$$

The output SIR criterion is widely used in performance evaluation of source separation algorithms when original source signals or mixing systems are available [39]. Since

\begin{tabular}{|c|c|c|c|c|c|c|}
\hline \multirow{2}{*}{$T$} & \multicolumn{3}{|c|}{ Alpha digits } & \multicolumn{3}{|c|}{ Poet verses } \\
\hline & JADE-AV & GMM-AVSS & MLP-AVSS & JADE-AV & GMM-AVSS & MLP-AVSS \\
\hline 4 & 18.3 & 24.0 & 27.7 & 7.1 & 11.9 & 13.3 \\
\hline 8 & 28.7 & 29.5 & 35.1 & 22.9 & 19.4 & 21.4 \\
\hline 16 & 32.9 & 35.9 & 39.9 & 30.8 & 30.2 & 32.1 \\
\hline 32 & 37.1 & 39.6 & 43.8 & 36.3 & 36.5 & 38.5 \\
\hline
\end{tabular}

Table 3 Average output SIRs in decibels for $2 \times 2$ case 
Table 4 Average output SIRs in dB for $3 \times 3$ case

\begin{tabular}{|c|c|c|c|c|c|c|}
\hline \multirow{2}{*}{$\mathbf{T}$} & \multicolumn{3}{|c|}{ Alpha digits } & \multicolumn{3}{|c|}{ Poet verses } \\
\hline & JADE-AV & GMM-AVSS & MLP-AVSS & JADE-AV & GMM-AVSS & MLP-AVSS \\
\hline 8 & 18.2 & 19.9 & 23.8 & 11.0 & 9.0 & 10.1 \\
\hline 16 & 22.8 & 25.3 & 29.1 & 18.8 & 18.2 & 19.8 \\
\hline 32 & 27.1 & 28.7 & 31.6 & 25.7 & 25.4 & 26.7 \\
\hline
\end{tabular}

in our experiments, we perform batch-wise separation, the output SIR is averaged over all batches in the test set. It is worth to mention that in convolutive mixtures, the SIRs must be calculated up to an allowed arbitrary filtering of the sources. This can be accomplished, using the decomposition method of Vincent et al. [39].

\subsubsection{Separation in regular $N \times N$ mixtures}

In this experiment, we consider regular $N \times N$ mixtures with equal number of sources and sensors. Simulations are performed for mixtures of different sizes $N=2,3,5$ and separation performance in terms of output SIR (21) is presented. Experiments are conducted on the test set of both alpha-digits (Persian and English) and poet-verses (Persian) corpora (see Section 4.1 for corpus details). Each corpus consists of a pair of synchronous audio and visual streams of frames. From each corpus, 3,000 frames are exploited in separation simulations. The audio stream from test corpus is considered as the relevant source $s^{1}$ and for other $N-1$ sources, speech signals of the same length are used. These speech signals are selected from a supplementary corpus recorded from other speakers with the same sampling frequency.

Since the performance of GMM- and MLP-based AVSS methods is not uniform in different mixture matrices, we have conducted Monte-Carlo (MC) simulations with 20 different random mixing matrices for each mixture size $N \times N$. Table 2 summarizes the average mixed input SIR of each sensor with respect to $s^{1}$ in various mixtures. Input SIRs are also useful in analysing the gained SIR specially in degenerate mixtures where output results is very sensitive to chosen mixing matrices. Mixing matrices are kept the same for both corpora. For each corpus and mixture size, input mean SIRs are obtained by calculating average on all the simulated random mixing matrices.

For each corpus and mixture size, speech source separation using, JADE-AV, GMM-AVSS and MLP-AVSS methods are conducted to all simulation matrices in order to estimate the de-mixing vectors. Then, the average output SIRs is calculated over all estimated de-mixing vectors and all batches of separated signals. Results are presented in Tables 3 (for $N=2$ ), 4 (for $N=3$ ) and 5 (for $N=5$ ). Since in this experiment, mixing matrices are squared and invertible, relatively high-output SIRs are achieved in all tested configurations. Analysis and comparison of results in terms of separation algorithms, batch integration size, mixture size and corpus reveals the following points:

Effect of discrete and continuous speech: The performance of all methods is higher on alpha-digits corpus compared to poet-verses. Alpha digits corpus is discrete and poet-verses corpus is continuous. It is obvious that continuous speech is more complex for AV modeling since lip formations are not well expressed due to speech speed (co-articulation) and also since in continuous corpus there is much number of different words and phonetic contexts which increases the phonetic complexity.

Relative separation performance of methods: In lower mixture sizes $(N=2,3)$, MLP-AVSS method provides higher output SIRs relative to GMM-AVSS and both of them are superior to JADE-AV for alpha-digits corpus. In $N=3,5$ and for poet-verses corpus, the performance enhancement gap between AVSS methods and JADE-AV is reduced. In this case, performance gain of GMM-AVSS is marginal and some times worst relative to JADE-AV. The superiority of MLP-AVSS relative to GMM-AVSS is consistent with classification accuracies of MLP and GMM-based AV models presented in Section 5.1.

Effect of batch integration time (T): The performance of all methods increases with increasing the number of frames in each batch. Increasing the integration time enhances accuracy of contrast functions (6) and (8) (see Section 5.1) and also reduces spurious local minima in the optimization landscape. For JADE-AV algorithm, in addition to improved accuracy of AV contrast, increasing

Table 5 Average output SIRs in dB for $5 \times 5$ case

\begin{tabular}{|c|c|c|c|c|c|c|}
\hline \multirow{2}{*}{$T$} & \multicolumn{3}{|c|}{ Alpha digits } & \multicolumn{3}{|c|}{ Poet verses } \\
\hline & JADE-AV & GMM-AVSS & MLP-AVSS & JADE-AV & GMM-AVSS & MLP-AVSS \\
\hline 8 & 4.9 & 11.9 & 13.8 & -1.2 & 0.4 & 2.2 \\
\hline 16 & 16.1 & 21.9 & 24.3 & 10.1 & 10.3 & 11.7 \\
\hline 32 & 20.3 & 26.2 & 29.4 & 17.0 & 17.2 & 19.1 \\
\hline
\end{tabular}


Table 6 Average output SIRs in dB for $2 \times 3$ case

\begin{tabular}{|c|c|c|c|c|c|c|}
\hline \multirow{2}{*}{$\mathbf{T}$} & \multicolumn{3}{|c|}{ Alpha digits } & \multicolumn{3}{|c|}{ Poet verses } \\
\hline & JADE-AV & GMM-AVSS & MLP-AVSS & JADE-AV & GMM-AVSS & MLP-AVSS \\
\hline 16 & 3.9 & 4.7 & 5.9 & -0.5 & -0.8 & 1.1 \\
\hline 32 & 4.1 & 5.2 & 7.4 & -0.6 & -0.7 & 2.9 \\
\hline 64 & 4.5 & 6.5 & 8.9 & 1.6 & 2.8 & 6.7 \\
\hline
\end{tabular}

integration time allows better estimates of higher order statistics of signals which affect separation quality of JADE algorithm. But recall that in real applications with nonstationary mixtures, there is a trade-off for increasing number of frames in each batch (see Section 5.1).

\subsubsection{Separation in degenerate $M \times N, M<N$ mixtures}

In this experiment, we performed Monte Carlo simulations with 20 random matrices of size $M \times N=2 \times 3$. Average mixed input SIRs of two channels on all simulated random matrices and for all test frames of each corpus is presented in the corresponding columns of Table 2. Like before, de-mixing matrices are estimated by running the proposed and baseline source separation methods. Results are presented in Table 6 .

In this case, the mixing matrices are degenerate and have not exact inverse. Hence, the perfect recovery of sources is not possible and SIRs are worse relative to regular $N \times N$ simulations. AVSS methods show slight improvements relative to JADE-AV methods. The performance of MLP-AVSS is again superior to GMM-AVSS as is predicted. In this experiment, results were highly dependent on mixing matrix. In some mixtures, output SIRs near to $10 \mathrm{~dB}$ were achieved while in some others negative output SIRs were observed.

\subsubsection{Separation of convolutive $2 \times 2$ mixtures}

In this experiment, we considered separation of $2 \times 2$ convolutive mixtures using methods described in Section 3.4. We generated random mixing systems for each filter size $(2 L+1)$ and simulated the mixtures. The separation was conducted with the same number of $(2 L+1)$ taps for each de-mixing filter. Due to the complexity of the convolutive problem, it is necessary to use large batch sizes. So we considered batches of $5 \mathrm{~s}$. Results in terms of output SIR are presented in Table 7.

For $L=0$, the mixture is instantaneous and separation is possible with high SIR. But for $L=2$ (filters with five taps) and for higher degree of mixing and de-mixing filters, the SIR decreases to about average $9 \mathrm{~dB}$ for audioonly method of [31] and $11 \mathrm{~dB}$ for the hybrid AV coherent and independent method proposed in Section 3.4.

\section{Conclusion}

In this paper, we proposed an improved AV association model using an MLP which exploits the dependency between AV frames and is superior to the existing GMM AV model. The MLP model makes efficient use of its parameters relative to the GMM model. Hence, unlike the GMM model, it can capture temporal dynamics from a limited context of frames around the current frame to enhance the coherency measure. We also proposed a hybrid criterion which exploits AV coherency together with normalized kurtosis as an independence measure and, based on that, moved toward a time-domain convolutive AVSS method. Experimental results for comparison of the methods are presented in terms of the relevant signal classification accuracy and also the separation output SIRs. Results, confirms the contribution of the proposed neural-based AV association model in enhancement of $\mathrm{AV}$ incoherency scores and hence in improvement of the separation SIRs compared to the existing GMM-based AVSS algorithm and the visually assisted ICA (JADE$\mathrm{AV}$ ) method. Also, results of the time-domain convolutive method, using hybrid AV criterion shows improvement compared to the reference audio-only method.

For visual parametrization part, we have used normalized PCA-projected (whitened) lip appearance features. PCA features do not need exact lip contour detection and hence require less computation compared to extraction of lip geometric (width and height) parameters. But it also has the drawback of being more sensitive to the speaker and segmentation of the lip region. The fitness of PCA features for AV modeling and AVSS task is justified by qualitative illustrations and numerical results. However, the proposed AVSS method is not coupled to the PCA visual features and it can be adopted with more robust and accurate visual features.

Although proposed model improves quality of AV modeling, but further enhancements is both required and predictable to make these methods applicable in more complex phonetic contexts and speaker-independent situations. AV relation is both non-linear and stochastic.

Table 7 Average output SIRs in dB for $2 \times 2$ convolutive case

\begin{tabular}{cccccccc}
\hline Method/L & $L=\mathbf{0}$ & $\boldsymbol{L}=\mathbf{2}$ & $\boldsymbol{L}=\mathbf{5}$ & $\boldsymbol{L}=\mathbf{1 0}$ & $\boldsymbol{L}=\mathbf{1 5}$ & $\boldsymbol{L}=\mathbf{2 0}$ & $\boldsymbol{L}=\mathbf{2 5}$ \\
\hline kurt $_{n}[31]$ & 36.2 & 10.1 & 9.6 & 9.7 & 8.9 & 8.5 & 8.6 \\
JaviCA (14) & 43.5 & 11.7 & 10.9 & 11.5 & 10.9 & 10.7 & 10.8 \\
\hline
\end{tabular}

Results for various mixing/de-mixing filter sizes $(2 L+1)$ are presented. Batch size is about 5 seconds. 
GMM benefits from its capability in probabilistic modeling. But GMM fails to efficiently handle the non-linearity and temporal dependency. On the other hand, MLP seems to benefit from its relatively deep structure and efficient use of its parameters, but it does not truly consider stochastic property of AV relation. Further improvements may be gained by introducing a model which can efficiently handle both the non-linear and the stochastic relations of the two modalities as well as the temporal dependency. Also, it seems promising to consider more essential combinations of ICA- and AV coherency-based methods to jointly gain benefits of both informed and blind methods.

Finally, it is worth to mention that in this paper, we did not consider the inter-batch temporal dynamics of de-mixing vectors and separated signals. It is possible to adopt this temporal information for example using a Bayesian recursive filtering approach to improve the performance and speed of proposed methods. Also, it is possible to adaptively determine the working batch size based on the amount of inter-batch variations of the de-mixing vectors.

\section{Appendix}

Here, we derive the gradient $\frac{\partial \mathcal{Y}_{e}(k)}{\partial B}$ which is required in calculation of (11). The embedded acoustic vector $\mathcal{Y}_{e}(k)$ is composed from individual acoustic frames $\mathcal{Y}(k)$. So we first need to calculate the gradient $\frac{\partial \mathcal{Y}(k)}{\partial B}$ which is a Jacobian matrix of the size $k_{a} \times M$. Starting from (17) and (19), we have

$$
\begin{aligned}
\frac{\partial \mathcal{Y}(k)}{\partial B} & =\frac{\partial F(B \mathbf{X}(k))}{\partial B} \\
& =\frac{\partial \mathbf{W}_{a}^{T} \log \left(\operatorname{PSD}_{B \mathbf{X}(k)}\right)}{\partial B} \\
& =\mathbf{W}_{a}^{T}\left[\frac{\partial \mathrm{PSD}_{B \mathbf{X}(k)}}{\partial B} \oslash \operatorname{PSD}_{B \mathbf{X}(k)}\right]
\end{aligned}
$$

where $\left(\mathbf{W}_{a}^{T}\right)_{k_{a} \times n}$ is the PCA (whitening) transform, $\left(\operatorname{PSD}_{B \mathbf{X}(k)}\right)_{n \times 1}$ is the power spectral density vector of frame $k,\left(\frac{\partial \mathrm{PSD}_{B \mathbf{X}(k)}}{\partial B}\right)_{n \times M}$ is Jacobian of PSD vector with respect to $B$ and $\oslash$ is the element-wise operator which divides each column of the left matrix by the right vector. The Jacobian of PSD is also calculated as:

$$
\begin{aligned}
\frac{\partial \mathrm{PSD}_{B \mathbf{X}(k)}}{\partial B} & =\frac{\partial \frac{\mathrm{PS}_{B \mathbf{X}(k)}}{\Sigma_{i} \mathrm{PS}_{B \mathbf{X}(k)}^{i}}}{\partial B} \\
& =\frac{\frac{\partial \mathrm{PS}_{B \mathbf{X}(k)}}{\partial B} \Sigma_{i} \mathrm{PS}_{B \mathbf{X}(k)}^{i}-\mathrm{PS}_{B \mathbf{X}(k)} \Sigma_{i} \frac{\partial \mathrm{PS}_{B \mathbf{X}(k)}^{i}}{\partial B}}{\left(\Sigma_{i} \mathrm{PS}_{B \mathbf{X}(k)}^{i}\right)^{2}}
\end{aligned}
$$

where $\left(\mathrm{PS}_{B \mathbf{X}(k)}\right)_{n \times 1}$ is the power spectrum vector, $\left(\Sigma_{i} \mathrm{PS}_{B \mathbf{X}(k)}^{i}\right)_{1 \times 1}$ is sum of its elements, $\left(\frac{\partial \mathrm{PS}_{B \mathbf{X}(k)}}{\partial B}\right)_{n \times M}$ is Jacobian matrix of the PS vector with respect to $B$ and $\left(\Sigma_{i} \frac{\partial \mathrm{PS}_{B \mathbf{X}(k)}^{i}}{\partial B}\right)_{1 \times M}$ is the sum of rows of the Jacobian matrix. Finally, the Jacobian of PS is calculated as

$$
\begin{aligned}
\frac{\partial \mathrm{PS}_{B \mathbf{X}(k)}}{\partial B}= & \frac{\partial\left[(B \mathbf{X}(k)) \otimes(B \mathbf{X}(k))^{*}\right]^{T}}{\partial B} \\
= & \frac{\partial\left[\operatorname{Re}\{B \mathbf{X}(k)\}^{2}+\operatorname{Im}\{B \mathbf{X}(k)\}^{2}\right]^{T}}{\partial B} \\
= & 2[\operatorname{Re}\{\mathbf{X}(k)\} \otimes \operatorname{Re}\{B \mathbf{X}(k)\}]^{T} \\
& +2[\operatorname{Im}\{\mathbf{X}(k)\} \otimes \operatorname{Im}\{B \mathbf{X}(k)\}]^{T}
\end{aligned}
$$

A where $\operatorname{Re}\{$.$\} and \operatorname{Im}\{$.$\} are real and imaginary part$ operators. Equations 22, 23 and 24 are derived for the calculation of Jacobian of a single frame. In our MATLAB implementation, we have derived more complex matrix forms which allows calculation of Jacobian of multiple acoustic frames (i.e. all frames in a batch) using efficient vectorized computing. Having Jacobian of individual acoustic frames $\mathcal{Y}(k)$, we combine the theme to obtain the Jacobian of embedded acoustic vectors $\mathcal{Y}_{e}(k)$. This is done according to the definition of the embedding method $E$.

\section{Competing interests}

The authors declare that they have no competing interests.

\section{Acknowledgements}

Authors would like to say thanks to the anonymous reviewers for their attention which solved some important presentation issues and also for providing improving suggestions.

Received: 7 July 2013 Accepted: 18 February 2014 Published: 5 April 2014

\section{References}

1. Sumby WH, Pollack I, Visual contribution to speech intelligibility in noise. J. Acoust. Soc. Am. 26, 212-215 (1954)

2. Summerfield Q, Lipreading and audio-visual speech perception. Philos. Trans. R. Soc. Lond. B Biol. Sci. 335(1273), 71-78 (1992)

3. KW Grant, P-F Seitz, The use of visible speech cues for improving auditory detection of spoken sentences. J. Acoust. Soc. Am. 108, 1197 (2000)

4. H McGurk, J MacDonald, Hearing lips and seeing voices. Nature. 264(5588), 746-748 (1976)

5. J-L Schwartz, F Berthommier, C Savariaux, Seeing to hear better: evidence for early audio-visual interactions in speech identification. Cognition. 93(2), 69-78 (2004)

6. ED Petajan, Automatic lipreading to enhance speech recognition. (PhD thesis, University of Illinois, Illinois, 1984)

7. L Girin, J-L Schwartz, G Feng, Audio-visual enhancement of speech in noise. J. Acoust. Soc. Am. 109(6), 3007-3020 (2001)

8. S Deligne, G Potamianos, C Neti, Audio-visual speech enhancement with AVCDCN (AudioVisual Code-book Dependent Cepstral Normalization), in Proceedings of the ISCA International Conference on Spoken Language Processing (ICSLP'02) (ISCA, 2002), pp. 1449-1452

9. F Berthommier, Audiovisual speech enhancement based on the association between speech envelope and video features, in Proceedings of the ISCA European Conference on Speech Communication and Technology (EUROSPEECH'O3) (ISCA, 2002), pp. 1045-1048

10. R Goecke, G Potamianos, C Neti, Noisy audio feature enhancement using audio-visual speech data, in Proc. IEEE International Conference on Acoustics, Speech, and Signal Processing (ICASSP'02), vol. 2 (IEEE, 2002), pp. 2025-2028 
11. B Rivet, L Girin, C Jutten, Visual voice activity detection as a help for speech source separation from convolutive mixtures. Speech Communication. 49(7), 667-677 (2007)

12. D Sodoyer, J-L Schwartz, L Girin, J Klinkisch, C Jutten, Separation of audio-visual speech sources: a new approach exploiting the audio-visual coherence of speech stimuli. EURASIP J. Appl. Signal Process. 2002(1), 1164-1173 (2002)

13. S Rajaram, AV Nefian, TS Huang, Bayesian separation of audio-visual speech sources, in Proc. IEEE International Conference on Acoustics, Speech, and Signal Processing (ICASSP'04), vol. 5 (IEEE, 2004), pp. 657-661

14. D Sodoyer, L Girin, C Jutten, J-L Schwartz, Developing an audio-visual speech source separation algorithm. Speech Commun. 44(1), 113-125 (2004)

15. W Wang, D Cosker, Y Hicks, S Sanei, J Chambers, Video assisted speech source separation, in Proc. IEEE International Conference on Acoustics, Speech, and Signal Processing (ICASSP'05), vol. 5 (IEEE, 2005), p. 425

16. B Rivet, L Girin, C Jutten, Mixing audiovisual speech processing and blind source separation for the extraction of speech signals from convolutive mixtures. Audio, Speech, Lang. Process. IEEE Trans. 15(1), 96-108 (2007)

17. C Sigg, B Fischer, B Ommer, V Roth, J Buhmann, Nonnegative CCA for audiovisual source separation, in IEEE Workshop On Machine Learning for Signal Processing (MLSP'07) (IEEE, 2007), pp. 253-258

18. AL Casanovas, G Monaci, P Vandergheynst, R Gribonval, Blind audiovisual separation based on redundant representations, in IEEE International Conference on Acoustics, Speech, and Signal Processing (ICASSP'08) (IEEE, 2008), pp. 1841-1844

19. G Monaci, F Sommer, P Vandergheynst, Learning sparse generative models of audiovisual signals, in EURASIP European Signal Processing Conference (EUSIPCO '08), vol. 4 (EURASIP, 2008)

20. G Monaci, P Vandergheynst, FT Sommer, Learning bimodal structure in audio-visual data. Neural Netw. IEEE Trans. 20(12), 1898-1910 (2009)

21. AL Casanovas, G Monaci, P Vandergheynst, R Gribonval, Blind audiovisua source separation based on sparse redundant representations. Multimedia, IEEE Trans. 12(5), 358-371 (2010)

22. Y Liang, SM Naqvi, JA Chambers, Audio video based fast fixed-point independent vector analysis for multisource separation in a room environment. EURASIP J. Adv. Signal Process. 2012(1), 1-16 (2012)

23. Q Liu, W Wang, PJ Jackson, M Barnard, J Kittler, JA Chambers, Source separation of convolutive and noisy mixtures using audio-visual dictionary learning and probabilistic time-frequency masking. IEEE Trans. Signal Process. 61(22) (2013)

24. MS Khan, SM Naqvi, A Rehman, W Wang, JA Chambers, Video-aided model-based source separation in real reverberant rooms. IEEE Trans. Audio Speech Lang. Process. 21(9), 1900-1912 (2013)

25. AJ Bell, TJ Sejnowski, An information-maximization approach to blind separation and blind deconvolution. Neural Computat. 7(6), 1129-1159 (1995)

26. A Hyvärinen, E Oja, A fast fixed-point algorithm for independent component analysis. Neural Comput. 9(7), 1483-1492 (1997)

27. J-F Cardoso, High-order contrasts for independent component analysis. Neural Comput. 11(1), 157-192 (1999)

28. J Jeffers, M Barley, Speechreading (lipreading). (Charles C. Thomas Publisher, Springfield, Illinois, 1971)

29. L Cappelletta, N Harte, Phoneme-to-viseme mapping for visual speech recognition, in Proceedings of the International Conference on Pattern Recognition Applications and Methods (ICPRAM 2012) (IEEE, 2012), pp. 322-329

30. N Delfosse, P Loubaton, Adaptive blind separation of independent sources: a deflation approach. Signal Process. 45(1), 59-83 (1995)

31. JK Tugnait, Identification and deconvolution of multichannel linear non-Gaussian, processes using higher order statistics and inverse filter criteria. Signal Process. IEEE Trans. 45(3), 658-672 (1997)

32. V Zarzoso, P Comon, Robust independent component analysis by iterative maximization of the kurtosis contrast with algebraic optimal step size. Neural Netw. IEEE Trans. 21(2), 248-261 (2010)

33. S Gazor, W Zhang, Speech probability distribution. Signal Process. Lett. IEEE. 10(7), 204-207 (2003)

34. I Tashev, A Acero, Statistical modeling of the speech signal, in Proc. Intl. Workshop on Acoustic, Echo, and Noise Control (IWAENC 2010) (IEEE, 2010)

35. J Thomas, Y Deville, S Hosseini, Time-domain fast fixed-point algorithms for convolutive ICA. Signal Process. Lett. IEEE. 13(4), 228-231 (2006)
36. F Moayedi, A Kazemi, Z Azimifar, Hidden Markov model-unscented Kalman filter contour tracking: a multi-cue and multi-resolution approach, in Iranian Conference on Machine Vision and Image Processing (MVIP 2010) (IEEE, Piscataway, 2010), pp. 1-6

37. AP Dempster, NM Laird, DB Rubin, Maximum likelihood from incomplete data via the EM algorithm. J. R. Stat. Soc. Series B (Methodological). 39(1), $1-38$ (1977)

38. MT Hagan, M Menhaj, Training feed-forward networks with the Marquardt algorithm. IEEE Trans. Neural Netw. 5(6), 989-993 (1994)

39. E Vincent, R Gribonval, C Févotte, Performance measurement in blind audio source separation. Audio, Speech, Lang. Process. IEEE Trans. 14(4), 1462-1469 (2006)

\section{doi:10.1186/1687-6180-2014-47}

Cite this article as: Kazemi et al:: Audio visual speech source separation via improved context dependent association model. EURASIP Journal on Advances in Signal Processing 2014 2014:47.

\section{Submit your manuscript to a SpringerOpen ${ }^{\odot}$ journal and benefit from:}

- Convenient online submission

Rigorous peer review

- Immediate publication on acceptance

- Open access: articles freely available online

- High visibility within the field

- Retaining the copyright to your article

Submit your next manuscript at $>$ springeropen.com 